\title{
On Extensions of Extended Gauss Hypergeometric Function
}

\author{
Ahmed Ali Atash ${ }^{1 *}$, Salem Saleh Barahmah ${ }^{1}$, Maisoon Ahmed Kulib
}

\section{Abstract}

The aim of this paper is to introduce a new extensions of extended Gauss hypergeometric function. Certain integral representations, transformation and summation formulas for extended Gauss hypergeometric function are presented and some special cases are also discussed.

Keywords: Extended hypergeometric function, Integral representations, Mittag-Leffler function, Summation formulas, Transformation formulas

2010 AMS: 33B15, 33C05, 33C20

\section{${ }^{1}$ Department of Mathematics, Aden University, Aden, Yemen}

*Corresponding author: ah-a-atash@hotmail.com

Received: 6 April 2019, Accepted: 26 July 2019, Available online: 30 September 2019

\section{Introduction}

The classical Beta function $B(x, y)$ is defined by:

$$
B(x, y)=\left\{\begin{array}{cl}
\int_{0}^{1} t^{x-1}(1-t)^{y-1} d t & , \quad(\operatorname{Re}(x)>0, \operatorname{Re}(y)>0) \\
\frac{\Gamma(x) \Gamma(y)}{\Gamma(x+y)} & , \quad \operatorname{Re}(x)>0, \operatorname{Re}(y)>0
\end{array}\right.
$$

where $\Gamma(x)$ is the familiar Gamma function defined by

$$
\Gamma(x)=\int_{0}^{\infty} t^{x-1} e^{-t} d t, \quad(\operatorname{Re}(x)>0) .
$$

The generalized hypergeometric function ${ }_{p} F_{q}$ with $p$ numerator parameters and $q$ denominator parameters is defined by (see [1])

$$
{ }_{p} F_{q}\left[\begin{array}{cc}
a_{1}, \ldots, a_{p} & ; \\
b_{1}, \ldots, b_{q} & ;
\end{array}\right]=\sum_{n=0}^{\infty} \frac{\left(a_{1}\right)_{n} \ldots\left(b_{p}\right)_{n}}{\left(a_{1}\right)_{n} \ldots\left(b_{q}\right)_{n}} \frac{z^{n}}{n !}
$$

where $(\lambda)_{n}$ is the well-known Pochhammer symbol. The case $p=2$ and $q=1$ of (1.2), yields the Gauss's hypergeometric function ${ }_{2} F_{1}(z)$. 
The Kampé de Fériet function of two variables $F_{l: m ; n}^{p: q ; k}[x, y]$ is defined by (see[1])

$$
F_{l: m ; n}^{p: q ; k}\left[\begin{array}{l}
\left(a_{p}\right):\left(b_{q}\right) ;\left(c_{k}\right) ; \\
\left(e_{l}\right):\left(f_{m}\right) ;\left(g_{n}\right) ;
\end{array}\right], y=\sum_{r, s=0}^{\infty} \frac{\prod_{j=1}^{p}\left(a_{j}\right)_{r+s} \prod_{j=1}^{q}\left(b_{j}\right)_{r} \prod_{j=1}^{k}\left(c_{j}\right)_{s}}{\prod_{j=1}^{l}\left(e_{j}\right)_{r+s} \prod_{j=1}^{m}\left(f_{j}\right)_{r} \prod_{j=1}^{n}\left(g_{j}\right)_{s}} \frac{x^{r}}{r !} \frac{y^{s}}{s !} .
$$

In 1903, Gosta Mittag-Leffler [2] introduced the function $E_{\alpha}(z)$ defined as:

$$
E_{\alpha}(z)=\sum_{n=0}^{\infty} \frac{z^{n}}{\Gamma(\alpha n+1)}, \quad \alpha>0, z \in \mathbb{C} .
$$

In 1905, Wiman [3] defined the generalized Mittag-Leffler function $E_{\alpha, \beta}(z)$ as follows:

$$
E_{\alpha, \beta}(z)=\sum_{n=0}^{\infty} \frac{z^{n}}{\Gamma(\alpha n+\beta)}, \quad(\alpha, \beta \in \mathbb{C}, \operatorname{Re}(\alpha)>0, \operatorname{Re}(\beta)>0) .
$$

Afterward, Prabhakar [4] defined the generalized Mittag-Leffler function $E_{\alpha, \beta}^{\gamma}(z)$ as follows:

$$
E_{\alpha, \beta}^{\gamma}(z)=\sum_{n=0}^{\infty} \frac{(\gamma)_{n}}{\Gamma(\alpha n+\beta)} \frac{z^{n}}{n !}, \quad(\alpha, \beta, \gamma \in \mathbb{C}, \operatorname{Re}(\alpha)>0, \operatorname{Re}(\beta)>0, \operatorname{Re}(\gamma)>0) .
$$

Clearly,

$$
E_{\alpha, \beta}^{1}=E_{\alpha, \beta}(z), \quad E_{\alpha, 1}^{1}=E_{\alpha}(z), \quad E_{1,1}^{1}=E_{1}(z)=e^{z} .
$$

In recent years, some extensions of Beta function and Gauss hypergeometric function have been considered by several authors (see $[5,6,7,8,9,10,11])$.

The following extended Beta function and extended Gauss hypergeometric function are introduced by Chaudhry et al. [12] and Chaudhry et al. [13] respectively:

$$
B(x, y ; p)=\int_{0}^{1} t^{x-1}(1-t)^{y-1} \exp \left(\frac{-p}{t(1-t)}\right) d t, \quad(\operatorname{Re}(p)>0, \operatorname{Re}(x)>0, \operatorname{Re}(y)>0)
$$

and

$$
F_{p}(a, b ; c ; z)=\sum_{n=0}^{\infty} \frac{B_{p}(b+n, c-b)}{B(b, c-b)}(a)_{n} \frac{z^{n}}{n !}, \quad(\operatorname{Re}(c)>\operatorname{Re}(b)>0, p \geq 0) .
$$

Choi et al. [14] introduced the extended Beta and extended Gauss hypergeometric functions as follows:

$$
B(x, y ; p ; q)=\int_{0}^{1} t^{x-1}(1-t)^{y-1} \exp \left(\frac{-p}{t}-\frac{q}{(1-t)}\right) d t, \quad(\operatorname{Re}(p)>0, \operatorname{Re}(q)>0)
$$

and

$$
F_{p, q}(a, b ; c ; z)=\sum_{n=0}^{\infty} \frac{B_{p, q}(b+n, c-b)}{B(b, c-b)}(a)_{n} \frac{z^{n}}{n !}, \quad(\operatorname{Re}(c)>\operatorname{Re}(b)>0, p, q \geq 0) .
$$

Rahman et al. [15] introduced the following extensions of (1.4) and (1.5) as follows:

$$
B_{p, q}^{\alpha}(x, y)=\int_{0}^{1} t^{x-1}(1-t)^{y-1} E_{\alpha}\left(-\frac{p}{t}\right) E_{\alpha}\left(-\frac{q}{(1-t)}\right) d t, \quad(\operatorname{Re}(\alpha)>0, p, q \geq 0)
$$

and

$$
F_{p, q}^{\alpha}(a, b ; c ; z)=\sum_{n=0}^{\infty} \frac{B_{p, q}^{\alpha}(b+n, c-b)}{B(b, c-b)}(a)_{n} \frac{z^{n}}{n !}, \quad(\operatorname{Re}(c)>\operatorname{Re}(b)>0, \operatorname{Re}(\alpha)>0, p, q \geq 0) .
$$


Further generalizations of (1.6) are introduced by Atash et al. [16] and Barahmah [17] as follows:

$$
B_{p, q}^{(\alpha, \beta)}(x, y)=\int_{0}^{1} t^{x-1}(1-t)^{y-1} E_{\alpha, \beta}\left(-\frac{p}{t}\right) E_{\alpha, \beta}\left(-\frac{q}{(1-t)}\right) d t, \quad(\operatorname{Re}(\alpha)>0, \operatorname{Re}(\beta)>0, p, q \geq 0)
$$

and

$$
B_{p, q}^{(\alpha, \beta, \gamma)}(x, y)=\int_{0}^{1} t^{x-1}(1-t)^{y-1} E_{\alpha, \beta}^{\gamma}\left(-\frac{p}{t}\right) E_{\alpha, \beta}^{\gamma}\left(-\frac{q}{(1-t)}\right) d t, \quad(\operatorname{Re}(\alpha)>0, \operatorname{Re}(\beta)>0, \operatorname{Re}(\gamma)>0, p, q \geq 0) .
$$

In the present paper, we aim to introduce new extensions for extended Gauss hypergeometric function by using (1.7) and (1.8) as follows:

$$
F_{p, q}^{(\alpha, \beta)}(a, b ; c ; z)=\sum_{n=0}^{\infty}(a)_{n} \frac{B_{p, q}^{(\alpha, \beta)}(b+n, c-b)}{B(b, c-b)} \frac{z^{n}}{n !}, \quad(\operatorname{Re}(c)>\operatorname{Re}(b)>0, \operatorname{Re}(\alpha)>0, \operatorname{Re}(\beta)>0, p, q \geq 0)
$$

and

$$
\begin{aligned}
F_{p, q}^{(\alpha, \beta, \gamma)}(a, b ; c ; z)= & \sum_{n=0}^{\infty}(a)_{n} \frac{B_{p, q}^{(\alpha, \beta, \gamma)}(b+n, c-b)}{B(b, c-b)} \frac{z^{n}}{n !}, \\
& (\operatorname{Re}(c)>\operatorname{Re}(b)>0, \operatorname{Re}(\alpha)>0, \operatorname{Re}(\beta)>0, \operatorname{Re}(\gamma)>0, p, q \geq 0) .
\end{aligned}
$$

Clearly,

$$
F_{p, q}^{(\alpha, \beta, 1)}=F_{p, q}^{(\alpha, \beta)}, F_{p, q}^{(\alpha, 1,1)}=F_{p, q}^{\alpha}, F_{p, q}^{(1,1,1)}=F_{p, q}, F_{p, p}^{(1,1,1)}=F_{p}, F_{0,0}^{(1,1,1)}={ }_{2} F_{1} .
$$

Further, if we use (1.7) in (1.9) and (1.8) in (1.10), we have respectively the following integral representations:

$$
\begin{array}{r}
F_{p, q}^{(\alpha, \beta)}(a, b ; c ; z)=\frac{1}{B(b, c-b)} \int_{0}^{1} t^{b-1}(1-t)^{c-b-1}(1-z t)^{-\alpha} E_{\alpha, \beta}\left(-\frac{p}{t}\right) E_{\alpha, \beta}\left(-\frac{q}{(1-t)}\right) d t, \\
(\operatorname{Re}(c)>\operatorname{Re}(b)>0, \operatorname{Re}(\alpha)>0, \operatorname{Re}(\beta)>0, p, q \geq 0)
\end{array}
$$

and

$$
\begin{array}{r}
F_{p, q}^{(\alpha, \beta, \gamma)}(a, b ; c ; z)=\frac{1}{B(b, c-b)} \int_{0}^{1} t^{b-1}(1-t)^{c-b-1}(1-z t)^{-\alpha} E_{\alpha, \beta}^{\gamma}\left(-\frac{p}{t}\right) E_{\alpha, \beta}^{\gamma}\left(-\frac{q}{(1-t)}\right) d t, \\
(\operatorname{Re}(c)>\operatorname{Re}(b)>0, \operatorname{Re}(\alpha)>0, \operatorname{Re}(\beta)>0, \operatorname{Re}(\gamma)>0, p, q \geq 0) .
\end{array}
$$

\section{Transformation and summation formulas}

In this section, we present some transformation and summation formulas for extended Gauss hypergeometric function (1.10) as follows:

Theorem 2.1. For $(\operatorname{Re}(c)>\operatorname{Re}(b)>0, \operatorname{Re}(\alpha)>0, \operatorname{Re}(\beta)>0, \operatorname{Re}(\gamma)>0, p, q \geq 0)$, the following transformation formula holds true:

$$
F_{p, q}^{(\alpha, \beta, \gamma)}(a, b ; c ; z)=(1-z)^{-a} F_{q, p}^{(\alpha, \beta, \gamma)}\left(a, c-b ; c ; \frac{-z}{1-z}\right) .
$$

Proof. Replacing $t$ by $(1-t)$ in (1.11) and using the following result:

$$
(1-z(1-t))^{-a}=(1-z)^{-a}\left(1-\frac{z}{z-1} t\right)^{-a},
$$

we obtain

$$
F_{p, q}^{(\alpha, \beta, \gamma)}(a, b ; c ; z)=\frac{(1-z)^{-a}}{B(b, c-b)} \int_{0}^{1} t^{c-b-1}(1-t)^{b-1}\left(1-\frac{z}{z-1} t\right)^{-a} E_{\alpha, \beta}\left(-\frac{q}{t}\right) E_{\alpha, \beta}\left(-\frac{p}{1-t}\right) d t,
$$

which, by applying (1.11) yields the desired result.

Remark 2.2. Replacing $z$ by $1-\frac{1}{z}$ and $\frac{z}{1+z}$ in (2.1), we have respectively 


\section{Corollary 2.3.}

$$
F_{p, q}^{(\alpha, \beta, \gamma)}\left(a, b ; c ; 1-\frac{1}{z}\right)=z^{a} F_{q, p}^{(\alpha, \beta, \gamma)}(a, c-b ; c ; 1-z) .
$$

\section{Corollary 2.4.}

$$
F_{p, q}^{(\alpha, \beta, \gamma)}\left(a, b ; c ; \frac{z}{1+z}\right)=(1+z)^{a} F_{q, p}^{(\alpha, \beta, \gamma)}(a, c-b ; c ;-z) .
$$

Theorem 2.5. For $(\operatorname{Re}(c-a-b)>0, \operatorname{Re}(k)>0, \operatorname{Re}(\beta)>0, \operatorname{Re}(\gamma)>0, p, q \geq 0)$, the following summation formula holds true:

$$
\begin{aligned}
& F_{p, q}^{(k, \beta, \gamma)}(a, b ; c ; 1)=\frac{\Gamma(c) \Gamma(c-a-b)}{\Gamma(\beta) \Gamma(\beta) \Gamma(c-a) \Gamma(c-b)}
\end{aligned}
$$

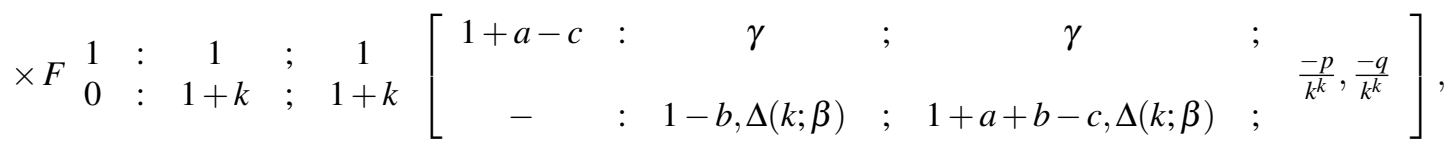

where $\Delta(k ; \beta)$ is $k$-tuple

$$
\frac{\beta}{k}, \frac{\beta+1}{k}, \ldots, \frac{\beta+k-1}{k} .
$$

Proof. From (1.11), we have

$$
F_{p, q}^{(\alpha, \beta, \gamma)}(a, b ; c ; 1)=\frac{1}{B(b, c-b)} \int_{0}^{1} t^{b-1}(1-t)^{c-a-b-1} E_{k, \beta}^{\gamma}\left(-\frac{p}{t}\right) E_{k, \beta}^{\gamma}\left(-\frac{q}{(1-t)}\right) d t .
$$

Applying (1.3) and interchanging the order of summation and integration and then using (1.1), we obtain

$$
F_{p, q}^{(\alpha, \beta, \gamma)}(a, b ; c ; 1)=\frac{\Gamma(c)}{\Gamma(\beta) \Gamma(\beta) \Gamma(c-b)} \times \sum_{r, s=0}^{\infty} \frac{(\gamma)_{r}(\gamma)_{s}(-p)^{r}(-q)^{s} \Gamma(b-r) \Gamma(c-a-b-s)}{(\beta)_{k r}(\beta)_{k s} r ! s !} .
$$

Now, using the following identities (see [1]):

$$
\frac{\Gamma(\alpha-n)}{\Gamma(\alpha)}=\frac{(-1)^{n}}{(1-\alpha)_{n}}
$$

and

$$
(\alpha)_{k n}=k^{k n} \prod_{j=1}^{k}\left(\frac{\alpha+j-1}{k}\right)_{n}, \quad n=1,2,3, \ldots
$$

we have

$$
\begin{aligned}
& F_{p, q}^{(k, \beta, \gamma)}(a, b ; c ; 1)=\frac{\Gamma(c) \Gamma(c-a-b)}{\Gamma(\beta) \Gamma(\beta) \Gamma(c-a) \Gamma(c-b)} \times \sum_{r, s=0}^{\infty} \frac{(1-c+a)_{r+s}(\gamma)_{r}(\gamma)_{s}(-p)^{r}(-q)^{s}}{k^{k r} \prod_{j=1}^{k}\left(\frac{\beta+j-1}{k}\right)_{r} k^{k s} \prod_{j=1}^{k}\left(\frac{\beta+j-1}{k}\right)_{s}(1-b)_{r}(1+a+b-c)_{s} r ! s !} \\
& =\frac{\Gamma(c) \Gamma(c-a-b)}{\Gamma(\beta) \Gamma(\beta) \Gamma(c-a) \Gamma(c-b)}
\end{aligned}
$$

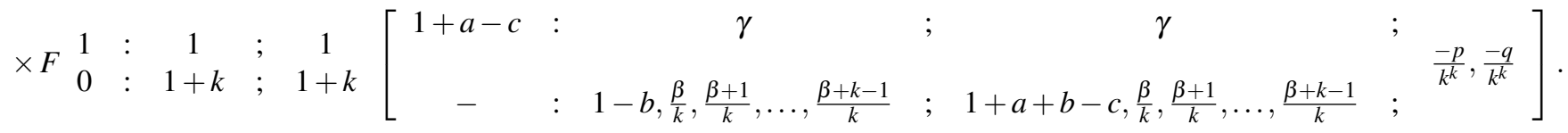

This completes the proof of (2.4).

Remark 2.6. Putting $a=-n$ in (2.4), we obtain 


\section{Corollary 2.7.}

$$
\begin{aligned}
& F_{p, q}^{(k, \beta, \gamma)}(-n, b ; c ; 1)=\frac{\Gamma(c) \Gamma(c+n-b)}{\Gamma(\beta) \Gamma(\beta) \Gamma(c+n) \Gamma(c-b)} \\
& \times F \begin{array}{ccccc}
1 & : & 1 & ; & 1 \\
0 & : & 1+k & ; & 1+k
\end{array}\left[\begin{array}{cccccc}
1-n-c & : & \gamma & ; & \gamma & ; \\
- & : & 1-b, \Delta(k ; \beta) & ; & 1-n+b-c, \Delta(k ; \beta) & ;
\end{array}\right.
\end{aligned}
$$

Remark 2.8. Putting $a=-n$ and $b=a+n$ in (2.4), we obtain

\section{Corollary 2.9.}

$$
\begin{aligned}
& F_{p, q}^{(k, \beta, \gamma)}(-n, a+n ; c ; 1)=\frac{\Gamma(c) \Gamma(c-a)}{\Gamma(\beta) \Gamma(\beta) \Gamma(c+n) \Gamma(c-a-n)} \\
& \times F \begin{array}{ccccc}
1 & : & 1 & ; & 1 \\
0 & : & 1+k & ; & 1+k
\end{array}\left[\begin{array}{cccccc}
1-n-c & : & \gamma & ; & \gamma & ; \\
- & : & 1-a-n, \Delta(k ; \beta) & ; & 1+a-c, \Delta(k ; \beta) & ;
\end{array}\right.
\end{aligned}
$$

Remark 2.10. Putting $a=-n$ and $b=1-b-n$ in (2.4), we obtain

\section{Corollary 2.11 .}

$$
\begin{aligned}
& F_{p, q}^{(k, \beta, \gamma)}(-n, 1-b-n ; c ; 1)=\frac{\Gamma(c) \Gamma(c+b-1+2 n)}{\Gamma(\beta) \Gamma(\beta) \Gamma(c+n) \Gamma(c+b-1-n)} \\
& \times F \begin{array}{cccccccc}
1 & : & 1 & ; & 1 \\
0 & : & 1+k & ; & 1+k
\end{array}\left[\begin{array}{ccccc}
1-n-c & : & \gamma & ; & \gamma \\
- & : & b+n, \Delta(k ; \beta) & ; & 2-b-c-2 n, \Delta(k ; \beta)
\end{array}\right]
\end{aligned}
$$

Theorem 2.12. For $(\operatorname{Re}(b)>0, \operatorname{Re}(k)>0, \operatorname{Re}(\beta)>0, \operatorname{Re}(\gamma)>0, p, q \geq 0)$, the following summation formula holds true:

$$
\begin{aligned}
& F_{p, q}^{(k, \beta, \gamma)}\left(-\frac{n}{2},-\frac{n}{2}+\frac{1}{2} ; b+\frac{1}{2} ; 1\right)=\frac{\Gamma\left(b+\frac{1}{2}\right) \Gamma(b+n)}{\Gamma(\beta) \Gamma(\beta) \Gamma\left(b+\frac{n}{2}\right) \Gamma\left(b+\frac{n}{2}+\frac{1}{2}\right)} \\
& \times F \begin{array}{ccccc}
1 & : & 1 & ; & 1 \\
0 & : & 1+k & ; & 1+k
\end{array}\left[\begin{array}{ccccc}
\frac{1}{2}-\frac{n}{2}-b & : & \gamma & \gamma & ; \\
- & : & (n / 2)+(1 / 2), \Delta(k ; \beta) & ; & 1-b-n, \Delta(k ; \beta)
\end{array}\right]
\end{aligned}
$$

where $\Delta(k ; \beta)$ is $k$-tuple

$$
\frac{\beta}{k}, \frac{\beta+1}{k}, \ldots, \frac{\beta+k-1}{k} .
$$

The proof of the Theorem 2.12 is similar to that of the Theorem 2.5. Therefore, we omit the details.

\section{Special cases}

(i) Setting $\beta=\gamma=1$ in (2.1), we get the following corrected formula given by Rahman et al. [15]

$$
F_{p, q}^{k}(a, b ; c ; z)=(1-z)^{-a} F_{q, p}^{k}\left(a, c-b ; c ; \frac{-z}{1-z}\right) .
$$

(ii) Setting $k=\beta=\gamma=1$ in (2.1), (2.2) and (2.3), we get a known transformation formulas of Choi et al. [14] for $F_{p, q}(a, b ; c ; z)$. (iii) Setting $k=\beta=\gamma=1, p=q$ in (2.1), we get a known transformation formula of Chaudhry et al. [13] for $F_{p}(a, b ; c ; z)$. 
(iv) Setting $k=\beta=\gamma=1, p=q=0$ in (2.1), we get Euler transformation [18, 1]).

(v) Setting $k=\beta=\gamma=1$ in (2.4), we get

$$
F_{p, q}^{(1,1,1)}(a, b ; c ; 1)=\frac{\Gamma(c) \Gamma(c-a-b)}{\Gamma(c-a) \Gamma(c-b)} \psi_{2}[1+a-c ; 1-b, 1+a+b-c ;-p,-q],
$$

where $\psi_{2}$ is the Humbert's confluent hypergeometric function [1].

By setting $p=q$ in (3.1) and using the result [1]

$$
\psi_{2}[a ; b, c ; x, x]={ }_{3} F_{3}\left[a, \frac{b+c}{2}, \frac{b+c-1}{2} ; b, c, b+c-1 ; 4 x\right],
$$

equation (3.1) reduces to

$$
F_{p, p}^{(1,1,1)}(a, b ; c ; 1)=\frac{\Gamma(c) \Gamma(c-a-b)}{\Gamma(c-a) \Gamma(c-b)} 2_{2}\left[\frac{a-c+1}{2}, \frac{a-c+2}{2} ; 1-b, 1+a+b-c ;-4 p\right] .
$$

Further, setting $p=0$ in (3.3), we get the well-known Gauss summation formula (see [18])

$$
F_{0,0}^{(1,1,1)}(a, b ; c ; 1)={ }_{2} F_{1}(a, b ; c ; 1)=\frac{\Gamma(c) \Gamma(c-a-b)}{\Gamma(c-a) \Gamma(c-b)} .
$$

(vi) Setting $k=\beta=\gamma=1$ in (2.5), we get

$$
F_{p, q}^{(1,1,1)}(-n, b ; c ; 1)=\frac{\Gamma(c) \Gamma(c+n-b)}{\Gamma(c+n) \Gamma(c-b)} \psi_{2}[1-n-c ; 1-b, 1-n+b-c ;-p,-q] .
$$

Further, setting $p=q=0$ in (3.4), we get a known result (see [18])

$$
F_{0,0}^{(1,1,1)}(-n, b ; c ; 1)={ }_{2} F_{1}(-n, b ; c ; 1)=\frac{(c-b)_{n}}{(c)_{n}} .
$$

(vii) Setting $k=\beta=\gamma=1$ in (2.6), we get

$$
F_{p, q}^{(1,1,1)}(-n, a+n ; c ; 1)=\frac{\Gamma(c) \Gamma(c-a)}{\Gamma(c+n) \Gamma(c-a-n)} \psi_{2}[1-n-c ; 1-a-n, 1+a-c ;-p,-q] .
$$

Further, setting $p=q=0$ in (3.5), we get a known result (see [18])

$$
F_{0,0}^{(1,1,1)}(-n, a+n ; c ; 1)={ }_{2} F_{1}(-n, a+n ; c ; 1)=\frac{(-1)^{n}(1+a-c)_{n}}{(c)_{n}} .
$$

(viii) Setting $k=\beta=\gamma=1$ in (2.7), we get

$$
F_{p, q}^{(1,1,1)}(-n, 1-b-n ; c ; 1)=\frac{\Gamma(c) \Gamma(b+c-1+2 n)}{\Gamma(c+n) \Gamma(b+c-1-n)} \psi_{2}[1-n-c ; b+n, 2-b-c-2 n ;-p,-q] .
$$

Further, setting $p=q=0$ in (3.6), we get a known result (see [18])

$$
F_{0,0}^{(1,1,1)}(-n, 1-b-n ; c ; 1)={ }_{2} F_{1}(-n, 1-b-n ; c ; 1)=\frac{(-1)^{n}(b+c-1)_{2 n}}{(c)_{n}(b+c-1)_{n}} .
$$

(viiii) Setting $k=\beta=\gamma=1$ in (2.8), we get

$$
F_{p, q}^{(1,1,1)}\left(-\frac{n}{2},-\frac{n}{2}+\frac{1}{2} ; b+\frac{1}{2} ; 1\right)=\frac{\Gamma\left(b+\frac{1}{2}\right) \Gamma(b+n)}{\Gamma\left(b+\frac{n}{2}\right) \Gamma\left(b+\frac{n}{2}+\frac{1}{2}\right)} \psi_{2}\left[\frac{1}{2}-\frac{n}{2}-b ; \frac{1}{2}+\frac{n}{2}, 1-b-n ;-p,-q\right],
$$

which for $p=q$ and using the result (3.2) reduces to

$$
F_{p, p}^{(1,1,1)}\left(-\frac{n}{2},-\frac{n}{2}+\frac{1}{2} ; b+\frac{1}{2} ; 1\right)=\frac{\Gamma\left(b+\frac{1}{2}\right) \Gamma(b+n)}{\Gamma\left(b+\frac{n}{2}\right) \Gamma\left(b+\frac{n}{2}+\frac{1}{2}\right)}{ }_{2} F_{2}\left[\frac{3}{4}-\frac{n}{4}-\frac{b}{2}, \frac{1}{4}-\frac{n}{4}-\frac{b}{2} ; \frac{1}{2}+\frac{n}{2}, 1-b-n ;-4 p\right] .
$$

Further, setting $p=0$ in (3.7) and using Legender's duplication formula (see [18])

$$
\Gamma(b) \Gamma\left(b+\frac{1}{2}\right)=2^{1-2 b} \sqrt{\pi} \Gamma(2 b),
$$

we get a known result (see [18])

$$
F_{0,0}^{(1,1,1)}\left(-\frac{n}{2},-\frac{n}{2}+\frac{1}{2} ; b+\frac{1}{2} ; 1\right)={ }_{2} F_{1}\left(-\frac{n}{2},-\frac{n}{2}+\frac{1}{2} ; b+\frac{1}{2} ; 1\right)=\frac{2^{n}(b)_{n}}{(2 b)_{n}} .
$$




\section{Acknowledgment}

The author is thankful to anonymous referees for useful comments and suggestions towards the improvement of this paper.

\section{References}

[1] H. M. Srivastava, P. W. Karlsson, Multiple Gaussian Hypergeometric Series, Halsted Press, New York, 1985.

[2] G. M. Mittag-Leffler, Sur la nouvelle function $E_{\alpha}(z)$, C. R. Acad. Sci. Paris, 137 (1903), 554-558.

[3] A. Wiman, Uber den fundamental Satz in der Theorie der Funktionen $E_{\alpha}(z)$, Acta Math., 29 (1905), 191-201.

[4] T. R. Prabhakar, A singuler integral equation with a generalized Mittag-Leffer function in the kernel, Yokohoma Math. J., 19 (1971), 7-15.

[5] A. A. Al-Gonah, W. K. Mohammed, A new extension of extended Gamma and Beta functions and their properties, J. Sci. Engrg. Res., 5(9) (2018), 257-270.

[6] P. Agarwal, Certain properties of the generalized Gauss hypergeometric functions, Appl. Math. Inform. Sci., 8(5)(2014), 2315-2320.

[7] P. Agarwal, J. Choi , S. Jain, Extended hypergeometric functions of two and three variables, Commun. Korean Math. Soc., 30(4) (2015), 403-414.

${ }^{[8]}$ M. Luo, G. V. Milovanovic, P. Agarwal, Some results on the extended beta and extended hypergeometric functions, Appl. Math. Comput., 248 (2014), 631-651.

[9] E. Özergin, M.A. Özarslan, A. Altin, Extension of gamma, beta and hypergeometric functions, J. Comp. and Appl. Math., 235 (2011), 4601-4610.

[10] P. I. Pucheta, A new extended Beta function, Int. J. Math. Appl, 5(3-C) (2017), 255-260.

[11] M. Shadab, S. Jabee, J. Choi, An extension of Beta function and its application, Far East J. Math. Sci., 103(1) (2018), 235-251.

[12] M. A. Chaudhry, A. Qadir, M. Rafique, S. M. Zubair, Extension of Euler's Beta function, J. Comput. Appl. Math., 78 (1997) 19-32.

[13] M. A. Chaudhry, A. Qadir, H. M.Srivastava, R. B. Paris, Extended hypergeometric and confluent hypergeometric function, Appl. Math. Comput., 159 (2004), 589-602.

[14] J. Choi, A. K. Rathie, R. K. Parmar, Extension of extended Beta, Hypergeometric and confluent hypergeometric functions, Honam Math. J., 36(2)(2014), 357-385.

[15] G. Rahman, G. Kanwal, K. S. Nisar, A. Ghaffar, A new extension of Beta and hypergeometric functions, (2018), doi:10.20944/preprints201801.0074.v1.

[16] A. A. Atash, S. S. Barahmah, M. A. Kulib, On a new extensions of extended Gamma and Beta functions, Int. J. Stat. Appl. Math., 3(6) (2018), 14-18.

[17] S. S. Barahmah, Further generalized Beta function with three parameters Mittag-Leffler function, Earthline J. Math. Sci., 1 (2019), 41-49.

[18] E. D. Rainville, Special Functions, The Macmillan Company, New York, (1960). 